\title{
Anesthesia and Decapitation Euthanasia
}

National Cancer Institute

\section{Source}

National Cancer Institute. Anesthesia and Decapitation Euthanasia. NCI Thesaurus. Code C90356.

A method of euthanization whereby a subject is anesthetized and the head is removed from the body. 